\title{
ON THE MECHANISM OF SURFACE ROUGHNESS WAKE FIELD EXCITATION*
}

\author{
S. Ratschow ${ }^{\S}$, T. Weiland, Darmstadt University of Technology, \\ FB 18, TEMF, Schloßgartenstrasse 8, 64289 Darmstadt, Germany. \\ M. Timm, CST GmbH, Büdinger Straße 2a, 64289 Darmstadt, Germany.
}

\begin{abstract}
In the manufacturing process beam pipes acquire a structure on their inner walls with a roughness depth in the order of typically $0.5 \mu \mathrm{m} \mathrm{rms}$. This surface structure is polarised by the beam and can be interpreted as an artificial dielectric.

The thin dielectric layer model shows, that for rough tubes there is exactly one synchronous mode, the so called "rough tube mode" (normally in the THz region), which couples to the beam. The existence of this mode was proven by $2 \mathrm{D}$ and $3 \mathrm{D}$ simulations.

In $2 \mathrm{D}$ the surface roughness is represented by irises. For a special kind of such 2D roughness, namely rectangular irises with random width and distance, there exists an easy way to explicitly calculate their equivalent dielectric properties. Equipped with these we calculate the wave number of the rough tube mode and contrast it to the wave number of the thin dielectric layer mode. In doing so, we present for this geometry a consistent mathematical formulation of rough surface wake fields.

In 3D simulations the surface roughness is represented by randomly distributed obstacles. We show two simulations with few obstacles. In one case the wake field turns out to be mainly inductive, whereas in the other case we find again the rough tube mode.
\end{abstract}

\section{INTRODUCTION}

Wake fields generated by the surface roughness of vacuum tubes, resulting from the manufacturing process, have been studied for quite some time: Future linacs will accelerate high bunch charges at very short bunch lengths. E.g. for the planned X-ray FEL of the TESLA project [1] a bunch charge of $1 \mathrm{nC}$ at $25 \mu \mathrm{m}$ rms length is projected. The Fourier spectrum of such a beam can drive wake fields up to the $\mathrm{THz}$ regime. The ongoing studies concentrate on whether the short range surface roughness wake fields generated by such bunches will significantly increase their energy width.

In [2] it was shown, using 2D simulations, that wake fields generated by surface corrugations (i.e. small irises on the inner tube surface) are similar to those of a smooth tube coated with a thin dielectric layer. In [2] the irises representing the surface roughness occupied about $50 \%$ of the surface area, and the results matched if the equivalent dielectric constant was chosen to be around $\varepsilon_{r}=2$. The

\footnotetext{
*Work supported in part by DESY, Hamburg, Germany.

${ }^{\S}$ ratschow@temf.tu-darmstadt.de
}

"thin dielectric layer model" of surface roughness was thoroughly investigated in [3] and related publications. The most important aspect of this model is, that in a rough tube exists a single synchronous "rough tube mode" (RTM) just as there is a "thin dielectric layer mode" (TDLM) in the coated tube.

In the following we review, how a special type of surface corrugation (rectangular irises of uniform height but random width and distance) can be viewed as an anisotropic dielectric layer. We calculate the RTM for this special type of roughness and compare it to the TDLM.

\section{THE THIN DIELECTRIC LAYER MODEL}

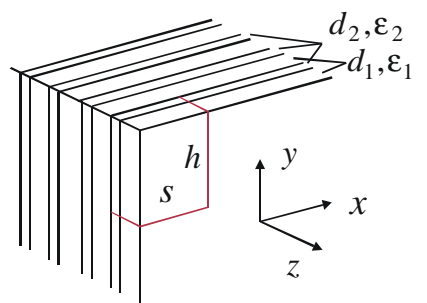

Fig.1: alternating layers of dielectrics.

\subsection{Averaging of Alternating Dielectric Layers}

Consider alternating layers of dielectrics as in Fig.1 with relative permittivities $\varepsilon_{1}$ and $\varepsilon_{2}$. Averaging of the material properties leads to an equivalent homogeneous anisotropic dielectric [4]. We use a quasi static approach, i.e. we assume that within the volumes, used for averaging, the fields do not change. The quasi static approach leads to correct results as long as the considered wavelengths are bigger than the typical period length.

In case of regular layers as shown in Fig. 1, it is sufficient to consider two adjacent layers. Tangential to the layers in the volume indicated in Fig. 1 two parallel capacities are replaced by an equivalent one, e.g. in $\mathrm{x}$ direction

$\frac{\varepsilon_{1} d_{1} h}{s}+\frac{\varepsilon_{2} d_{2} h}{s}=\frac{\varepsilon_{x}\left(d_{1}+d_{2}\right) h}{s} \Rightarrow \varepsilon_{x}=\frac{\varepsilon_{1} d_{1}+\varepsilon_{2} d_{2}}{d_{1}+d_{2}}$.

Normal to the layers the averaging process combines two serial capacities

$$
\frac{1}{\frac{\varepsilon_{1} h s}{d_{1}}}+\frac{1}{\frac{\varepsilon_{2} h s}{d_{2}}}=\frac{1}{\frac{\varepsilon_{z} h s}{d_{1}+d_{2}}} \Rightarrow \varepsilon_{z}=\frac{d_{1}+d_{2}}{\frac{d_{1}}{\varepsilon_{1}}+\frac{d_{2}}{\varepsilon_{2}}} .
$$

Obviously the result of this averaging, when carried out over appropriately large regions in space, does not depend 
on any periodicity of the layers. This explains, why the thin dielectric layer model applies equally well to periodic as to random surface perturbations.

\subsection{Conducting Materials}

If material 2 is a good conductor the same expressions Eqs. (1), (2) derived above can be used, for the complex dielectric constant

$$
\varepsilon_{2} \approx \frac{\kappa_{2}}{i \omega},
$$

where $\kappa_{2}$ denotes the conductivity of material 2. $\kappa_{2}$ will typically be such that

$$
\left|\varepsilon_{2}\right| \succ \succ\left|\varepsilon_{1}\right| \text {, }
$$

which immediately reduces Eq. (2) to

$$
\varepsilon_{z} \approx \varepsilon_{1} \frac{d_{1}+d_{2}}{d_{1}} \text {. }
$$

Eq. (5) reflects, that $E_{z}$ is confined to the layers of material 1 between the conducting sheets, while the latter are on fixed potentials. In order to reach the required voltage difference the field in the layers of material 1 is proportionally higher.

The parallel permittivities according to Eq. (1) will be almost purely imaginary

$$
\varepsilon_{x, y} \approx \frac{\kappa_{2}}{i \omega} \frac{d_{2}}{d_{1}+d_{2}} .
$$

This result tells us that parallel electric fields will mainly not penetrate the layers between the conducting sheets.

Next we turn to the magnetic fields. We remark, that the parallel magnetic fields in the layers 1 will neither be reduced nor amplified. But there are no magnetic fields in the conducting sheets 2 . Thus, when averaging over the whole space, we require, that

$$
\mu_{x, y}=\mu_{1} \frac{d_{1}}{d_{1}+d_{2}} .
$$

The results so far have been derived in Cartesian coordinates. If the surface layer is small compared to the radius of the beam tube, they can be used the same for circular cylindrical co-ordinates.

In this case radial electric fields induce charges on the irises. If the induced charges depend on $z$ there will be longitudinal electric fields. According to our quasi static approach, they are concentrated in the gaps. This justifies Eq. (5) also for this case.

\section{CALCULATION OF THE WAVE NUMBER OF THE ROUGH TUBE MODE}

\subsection{The Rough Tube Mode}

We calculate the fundamental RTM in a tube with circular cross section in cylindrical co-ordinates. The RTM is a synchronous $\left(k_{z}=k_{0}=\omega / c\right)$ TM-mode with the following field configuration:

$$
\partial / \partial \varphi \equiv 0, E_{\varphi}=0, H_{z}=0, H_{\rho}=0 .
$$

We insert Eq. (8) into Maxwell's equations in Gaussian units

$$
\operatorname{curl} \vec{H}=i k_{0} \varepsilon \vec{E} ; \quad \operatorname{curl} \vec{E}=-i k_{0} \mu \vec{H},
$$

and find in the usual way for $H_{\varphi}$ (keeping in mind, that $\varepsilon$ and $\mu$ are tensors in our case)

$$
\left(\frac{\partial^{2}}{\partial \rho^{2}}+\frac{1}{\rho} \frac{\partial}{\partial \rho}-\frac{1}{\rho^{2}}+\frac{\varepsilon_{z}}{\varepsilon_{\rho}} \frac{\partial^{2}}{\partial z^{2}}+k_{0}^{2} \mu_{\varphi} \varepsilon_{z}\right) H_{\varphi}=0 .
$$

We solve this equation separately first in the inner vacuum region and second in the surface layer.

a) Within the vacuum $\varepsilon_{z}=\varepsilon_{\rho}=\mu_{\varphi}=1$, and

$$
H_{\varphi}=C \rho e^{-i k_{0} z} ; \quad E_{\rho}=H_{\varphi} ; \quad E_{z}=\frac{2}{i k_{0}} C e^{-i k_{0} z}
$$

solves the system of equations Eqs. (9), (10). We remark that this is a synchronous mode as wanted, that it has finite field values at the origin and that the longitudinal electric field does not depend on $\rho$.

b) Turning to the thin surface layer, we use the ansatz

$$
H_{\varphi}=f(\rho) e^{-i k_{0} z}
$$

which reduces Eq. (10) to

$$
\left(\frac{\partial^{2}}{\partial \rho^{2}}+\frac{1}{\rho} \frac{\partial}{\partial \rho}-\frac{1}{\rho^{2}}+K^{2}\right) f(\rho)=0
$$

with $K^{2}=k_{0}^{2}\left(\mu_{\varphi} \varepsilon_{z}-\varepsilon_{z} / \varepsilon_{\rho}\right) \neq 0$. Bessel's equation Eq. (13) is well known to have the following general solution, using Bessel $J_{1}$ and Neumann $Y_{1}$ functions

$$
f(\rho)=A J_{1}(K \rho)+B Y_{1}(K \rho) .
$$

Our solution is therefore

$$
\begin{aligned}
& H_{\varphi}=\left(A J_{1}(K \rho)+B Y_{1}(K \rho)\right) e^{-i k_{0} z} ; \quad E_{\rho}=\left(1 / \varepsilon_{\rho}\right) H_{\varphi} \\
& E_{z}=\frac{K}{i k_{0} \varepsilon_{z}}\left(A J_{0}(K \rho)+B Y_{0}(K \rho)\right) e^{-i k_{0} z}
\end{aligned}
$$

\subsection{Boundary and Continuity Conditions}

Let us first fix the dimensions of the tube. Let $\delta$ be the thickness of the surface layer (height of the irises or thickness of dielectric), let the surface layer start at $\rho_{1}=R$, then the outer perfectly conducting wall of the tube is at $\rho_{2}=R+\delta$.

The boundary condition $E_{z}\left(\rho_{2}\right)=0$ relates the constants $A, B$ yielding

$$
A=D Y_{0}\left(K \rho_{2}\right) ; \quad B=-D J_{0}\left(K \rho_{2}\right) .
$$

The continuity condition for tangential $E_{z}\left(\rho_{1}\right)$ reads

$\frac{2}{i k_{0}} C=\frac{K D}{i k_{0} \varepsilon_{z}}\left(Y_{0}\left(K \rho_{2}\right) J_{0}\left(K \rho_{1}\right)-J_{0}\left(K \rho_{2}\right) Y_{0}\left(K \rho_{1}\right)\right)$.

The continuity condition for tangential $H_{\varphi}\left(\rho_{1}\right)$ is

$$
C \rho_{1}=D\left(Y_{0}\left(K \rho_{2}\right) J_{1}\left(K \rho_{1}\right)-J_{0}\left(K \rho_{2}\right) Y_{1}\left(K \rho_{1}\right)\right) \text {. }
$$

Taking the quotient of Eqs. (17), (18), to get rid of the constants 


$$
\frac{2 \varepsilon_{z}}{\rho_{1} K}=\frac{Y_{0}\left(K \rho_{2}\right) J_{0}\left(K \rho_{1}\right)-J_{0}\left(K \rho_{2}\right) Y_{0}\left(K \rho_{1}\right)}{Y_{0}\left(K \rho_{2}\right) J_{1}\left(K \rho_{1}\right)-J_{0}\left(K \rho_{2}\right) Y_{1}\left(K \rho_{1}\right)} .
$$

This non linear determining equation for $K$ can in general only be solved numerically. But if $K \rho_{1}, K \rho_{2} \succ \succ 1$ the asymptotic expressions for the Bessel and Neumann functions $J_{0}(x) \approx\left(\frac{2}{\pi x}\right)^{\frac{1}{2}} \cos \left(x-\frac{\pi}{4}\right)$ etc. can be used. After an easy calculation we find

$$
\frac{2 \varepsilon_{z}}{\rho_{1} K}=\frac{\sin (K \delta)}{\cos (K \delta)+\frac{1}{2 K \sqrt{\rho_{1} \rho_{2}}} \sin (K \delta)} .
$$

If now furthermore $K \delta \prec \prec 1$, which actually specifies, what we mean by a "thin" layer, the right hand side of Eq. (20) can be approximated by $\approx \tan (K \delta) \approx K \delta$ to yield

$$
K^{2}=\frac{2 \varepsilon_{z}}{R \delta} .
$$

The final step is to relate $K$, the wave number in the surface layer in radial direction, to $k_{0}$, the synchronous wave number in the vacuum, via

$$
K^{2}=k_{0}^{2}\left(\mu_{\varphi} \varepsilon_{z}-\varepsilon_{z} / \varepsilon_{\rho}\right) .
$$

At this step the RTM and the TDLM behave differently.

a) For the RTM $\left|\varepsilon_{\rho}\right| \succ \succ\left|\varepsilon_{z}\right|$, and we may safely neglect the second summand. We are left with $K^{2}=k_{0}^{2}$ and the RTM wave number is

$$
k_{0}^{2}=\frac{2 \varepsilon_{z}}{R \delta}=\frac{2}{R \delta} \frac{d_{1}+d_{2}}{d_{1}} .
$$

The last formula was used in [3] to interpret simulation results. It was found to be an agreeable approximation. Deviations naturally were found for distances between the irises longer than the wavelength of the RTM and for iris thickness' bigger than the bunch length.

b) For the TDLM we have $\varepsilon_{z}=\varepsilon_{\rho}=: \varepsilon_{r}$ and $\mu_{\varphi}=1$ which leads to the TDLM wave number

$$
k_{0}^{2}=\frac{2}{R \delta} \frac{\varepsilon_{r}}{\varepsilon_{r}-1} .
$$

The difference between the two wave numbers is caused by the direction and the speed of the wave in the surface layer. The RTM, as derived here, is directed purely radial in the gaps and its speed is equal to $c$.

\section{SIMULATION OF 3D OBSTACLES}

The thin dielectric layer model described above is a $2 \mathrm{D}$ theory, averaging the rough surface in longitudinal direction. Furthermore the thin dielectric layer model is limited to situations where the distance between the irises is small. The effective consequence of this is, that no radial electric fields penetrate the gaps. This condition is not fulfilled for the following examples. Still it is worthwhile to ask whether a $2 \mathrm{D}$ theory will be able to describe "real world" rough tube wake fields.

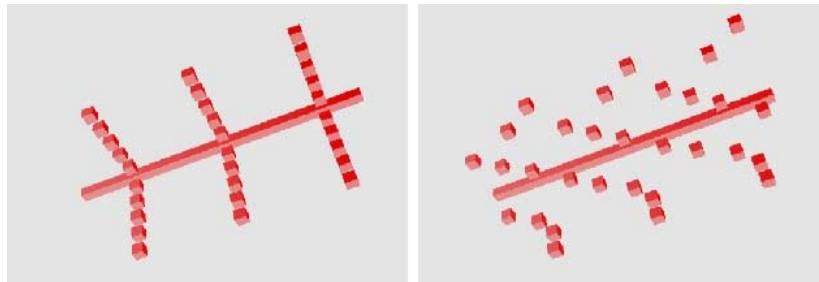

Fig.2: models of surface roughness with longitudinally equidistant cubes. Period length $1 \mathrm{~mm}$, cube side length

$0.1 \mathrm{~mm}$, transverse distance between cubes $0.1 \mathrm{~mm}$.

a) all the same z-position b) random z-position.

We simulated with Mafia T3 module in a true 3D environment cubes, equally spaced in $z$ direction, once all with the same $z$ position, once with random $z$ positions.

In the regular case the wake field tends to be inductive. The big distance between the "irises" makes them appear as single obstacles. In case of random longitudinal position we find again a RTM with a rather strong damping caused by decoherence effects. Obviously the longitudinal distance between the obstacles is effectively reduced by the random positioning.
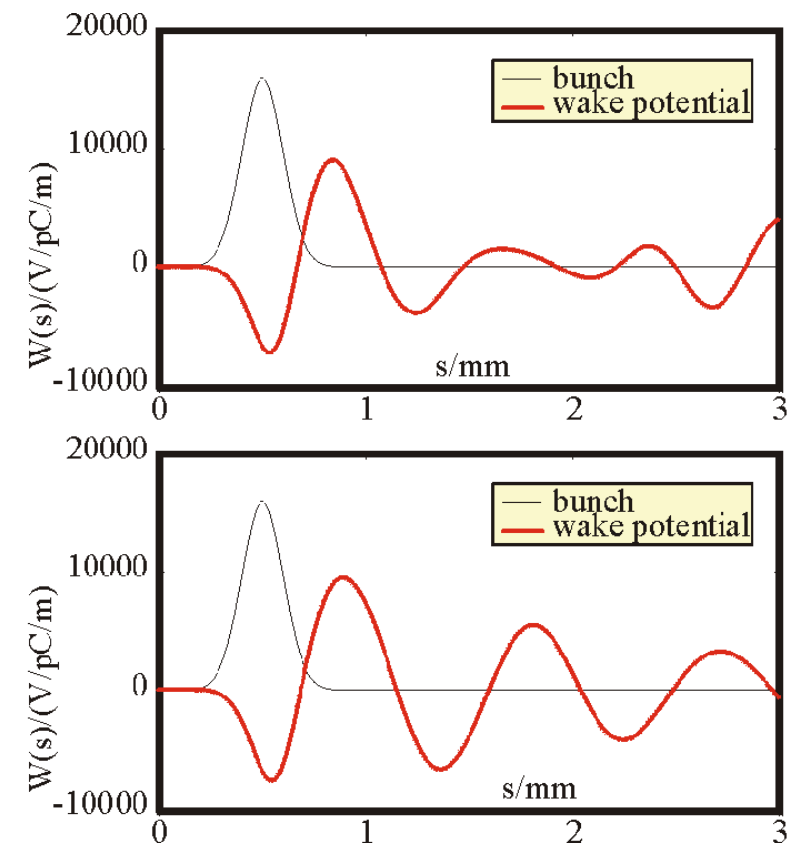

Fig. 3: longitudinal wake potentials for the geometries shown in Fig. (2). case a) above. case b) below.

\section{REFERENCES}

[1] TESLA Technical Design Report, DESY 2001-011, Part II (p. 289), Hamburg 2001.

[2] A. Novokhatski, A. Mosnier, "Wake Fields of Short Bunches in the Canal Covered With Thin Dielectric Layer", PAC'97, pp. 1444-1446, Vancouver 1997.

[3] M. Timm, "Wake Fields of Short Ultra-Relativistic Electron Bunches", Der Andere Verlag, Osnabrück 2001, Darmstädter Dissertation D17.

[4] G. Piefke, "Feldtheorie III" pp. 313-345, BI, Mannheim, Wien, Zürich 1977. 